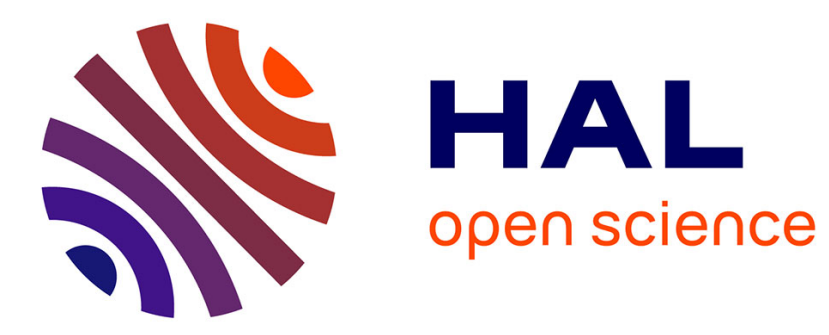

\title{
Étude des paramètres de l'autodiffusion de surface dans une direction donnée en émission de champ (tungstène)
}

\author{
J. Bardon, M. Drechsler
}

\section{To cite this version:}

J. Bardon, M. Drechsler. Étude des paramètres de l'autodiffusion de surface dans une direction donnée en émission de champ (tungstène). Revue de Physique Appliquée, 1974, 9 (6), pp.989-993. 10.1051/rphysap:0197400906098900 . jpa-00243870

\section{HAL Id: jpa-00243870 https://hal.science/jpa-00243870}

Submitted on 1 Jan 1974

HAL is a multi-disciplinary open access archive for the deposit and dissemination of scientific research documents, whether they are published or not. The documents may come from teaching and research institutions in France or abroad, or from public or private research centers.
L'archive ouverte pluridisciplinaire HAL, est destinée au dépôt et à la diffusion de documents scientifiques de niveau recherche, publiés ou non, émanant des établissements d'enseignement et de recherche français ou étrangers, des laboratoires publics ou privés. 


\title{
ÉTUDE DES PARAMÈTRES DE L'AUTODIFFUSION DE SURFACE DANS UNE DIRECTION DONNÉE EN ÉMISSION DE CHAMP (TUNGSTẼNE)
}

\author{
J. BARDON et M. DRECHSLER \\ Laboratoire des Mécanismes de la Croissance Cristalline, \\ Université d'Aix-Marseille III, Centre de Saint-Jérôme, \\ 13397 Marseille Cedex 4, France
}

(Reçu le 20 mars 1974, révisé le 17 juin 1974)

\begin{abstract}
Résumé. - Nous avons mis au point une méthode permettant de mesurer en microscopie électronique de champ la diffusivité $D_{0}$ et l'énergie d'activation $Q_{\text {s }}$ pour l'autodiffusion de surface. Cette méthode est une extension de celle où $Q_{\text {s }}$ est déterminé en observant l'évolution par diffusion de surface d'une forme de champ vers une forme d'équilibre.

Nous mesurons maintenant l'évolution de la géométrie du cristal, ce qui nous permet, en utilisant un calcul de Gjostein, de déterminer à la fois $Q_{\mathrm{s}}$ et $D_{0}$. La méthode permet de mesurer ces coefficients dans des directions (zones) déterminées. La mesure de $D_{0}$ et de $Q_{\mathbf{s}}$ a été effectuée pour le tungstène entre les faces $(110) \leftrightarrow(100)$ et $(211) \leftrightarrow(100)$. Les résultats obtenus $\left(34 \mathrm{~cm}^{2} / \mathrm{s} ; 3,8 \mathrm{eV}\right.$ et $0,3 \mathrm{~cm}^{2} / \mathrm{s}$; $3,1 \mathrm{eV})$ paraissent en accord avec les valeurs trouvées dans la littérature.
\end{abstract}

Abstract. - We describe a method of measuring the diffusivity $D_{0}$ and the activation energy $Q_{s}$ of surface self-diffusion by field emission microscopy. The method is an extension of those which determine $Q_{\mathrm{s}}$ by observing the change of shape of a tip crystal from a build-up form into an equilibrium form. A measurement of the evolution of the crystal geometry permits (using a calculation of Gjostein) the determination of $Q_{\mathrm{s}}$ as well as $D_{0}$. As a test, the $D_{0}$ and $Q_{\mathrm{s}}$ of tungsten are measured between $(110) \leftrightarrow(100)$ and $(211) \leftrightarrow(100)$. The results $\left(34 \mathrm{~cm}^{2} / \mathrm{s}, 3.8 \mathrm{eV}\right.$ and $\left.0.3 \mathrm{~cm}^{2} / \mathrm{s}, 3.1 \mathrm{eV}\right)$ are in reasonable agreement with known experimental and theoretical data. The method is relatively simple, permits measurements in definite crystallographic directions under extreme surface cleanliness and does not require a measurement of the profile or the cone angle of the tip.

1. Introduction. - La microscopie par émission de champ présente un grand intérêt pour l'étude de la diffusion de surface ; en effet, ce phénomène dépend dans de grandes proportions des impuretés pouvant contaminer la surface étudiée, or l'émission de champ permet un contrôle continu de la propreté de la surface et une décontamination aisée par chauffage de la pointe émettrice.

Il n'existe pas de méthode universelle de mesure de l'autodiffusion de surface parce que ce phénomène est assez complexe.

On peut d'abord considérer le déplacement d'adatomes individuels sur une face dense, mesurée d'abord en microscopie électronique de champ [1], puis en microscopie ionique de champ [2, 3]. Ce type d'autodiffusion ne conduit pas à un changement de la forme du cristal étudié.

L'autodiffusion de surface qui conduit à un changement de forme se passe suivant une ou plusieurs zones cristallographiques. Dans ce cas, il existe d'une part des mesures basées sur l'approximation d'une diffusion isotrope (mesure de l'augmentation du rayon de courbure moyen à l'apex d'une pointe [4, $5,6,7,8,9])$, d'autre part des études où l'énergie d'activation $Q_{\mathrm{s}}$ de cette diffusion dans une direction cristallographique est mesurée $[10,11]$ ou mesurable mais négligée $[12,13,14]$.

Il nous a semblé intéressant de développer une méthode qui permette de mesurer dans une direction donnée en microscopie électronique de champ non seulement $Q_{\mathrm{s}}$ mais aussi la diffusivité $D_{0}$, donc le coefficient d'autodiffusion de surface $D_{\mathrm{s}}$ qui sont liés par la relation :

$$
D_{\mathrm{s}}=D_{0} \mathrm{e}^{-\mathrm{Q}_{\mathrm{s}} / k T} .
$$

La méthode décrite ici est une extension de celle que Pichaud et Drechsler $[12,13]$ ont utilisée pour l'étude de l'influence des couches d'adsorption sur l'autodiffusion de surface où l'on mesure l'évolution d'une forme de champ à une forme d'équilibre.

Par rapport aux autres méthodes $[1,4,5,6]$ celle-ci présente l'ensemble des avantages suivants :

- le contrôle facile de l'état de la surface, soit 
sans adsorption, soit en présence d'une couche d'adsorption donnée,

- la possibilité de mesurer en gardant constant le profil moyen de la pointe,

- celle d'effectuer plusieurs mesures sans changement de la pointe,

- un contrôle du profil de la pointe et une mesure de son angle de cône (par microscopie de balayage par exemple) ne sont pas nécessaires,

- la possibilité d'utiliser un domaine de températures relativement basses car les transferts de masse sont très faibles.

2. Théorie. - La détermination du facteur $D_{0}$ nécessite la résolution complète de l'équation différentielle du quatrième ordre à laquelle conduisent les phénomènes de diffusion de surface. Cette résolution n'a pas, à notre connaissance, été effectuée pour la géométrie d'une pointe d'émission de champ. Le problème est très simplifié si l'on peut le ramener de trois dimensions à une seule. C'est ce qui a été fait par Gjostein [15] qui traite le cas d'un cylindre infini dont l'énergie de surface $\gamma$ et le rayon vecteur $R$ s'écartent peu de leurs valeurs moyennes $\gamma_{0}$ et $R_{0}$ (Fig. 1b).

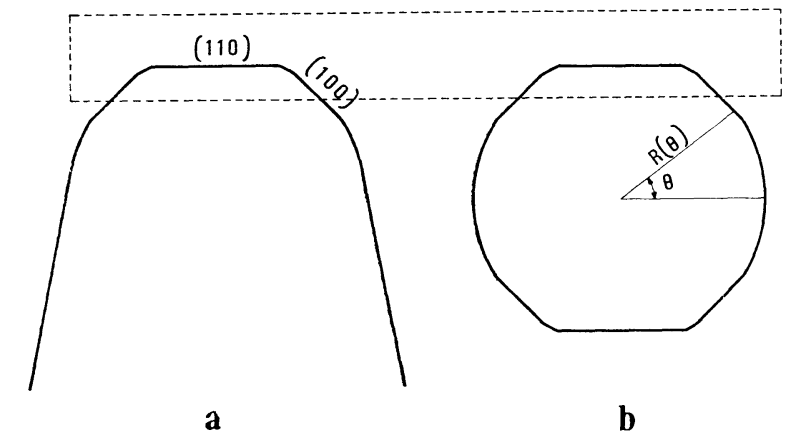

FIG. 1. - a) Forme de la pointe à un instant donné ; b) Modèle utilisé. La partie encadrée correspond à la partie utilisée pour les mesures.

Dans ces conditions, en utilisant

- deux développements en série de Fourier,

$$
\begin{aligned}
& \gamma(\theta)=\gamma_{0}\left(1+\sum_{m} \gamma_{m} \cos m \theta\right) \\
& R(\theta)=R_{0}\left(1+\sum_{m} R_{m} \cos m \theta\right)
\end{aligned}
$$

- les relations classiques donnant le flux de diffusion et le potentiel chimique sous leur forme monodimensionnelle [21]:

$$
\begin{aligned}
& J=\left(N_{0} D_{\mathrm{s}} / k T\right) \cdot \operatorname{grad}\left(\mu-\mu_{0}\right) \\
& \mu=\mu_{0}+\Omega / r_{0}\left(\gamma+\partial^{2} \gamma / \partial \theta^{2}\right)
\end{aligned}
$$

où $-\mu$ est le potentiel chimique,

- $N_{0}$ le nombre de sites par unité de surface,

- $\Omega$ le volume atomique,

- $D_{\mathrm{s}}$ le coefficient d'autodiffusion de surface,
- la relation exprimant la conservation du volume total

$$
\partial R / \partial t=\Omega / R \quad \partial J / \partial \theta
$$

où $t$ est le temps, on obtient :

$$
R_{m}(t)=\gamma_{m}+\left(R_{m}(0)-\gamma_{m}\right) \mathrm{e}^{-\left(B t / R_{0}^{4}\right) \cdot m^{2}\left(m^{2}-1\right)}
$$

avec

$$
B=\frac{\gamma N_{0} D_{\mathrm{s}} \Omega^{2}}{k T}
$$

Il est donc possible, connaissant l'évolution de la forme du cylindre en fonction du temps et de la température, d'en déduire $B$ donc $D_{0}$ et $Q_{\mathrm{s}}$.

Pour pouvoir appliquer ce calcul à une pointe émettrice, nous avons travaillé dans les conditions suivantes :

Nous effectuons les mesures dans un plan de section de la pointe pour lequel les variations du rayon de courbure local sont importantes, ce qui permet de supposer que la diffusion de surface s'effectue principalement dans ce plan. Les plans de section répondant à cette condition sont imposés par le processus anisotrope de build up.

La partie observée de la pointe est proche de l'apex, l'influence de la forme des parties éloignées de l'apex pourra donc être négligée.

Ces deux hypothèses permettent d'utiliser l'approximation cylindrique dans laquelle la partie observée de la pointe est représentée par une partie de la surface d'un cylindre infini (Fig. 1).

La procédure utilisée pour la détermination de $D_{0}$ et de $Q_{\text {s }}$ est la suivante :

- L'évolution en fonction du temps, par chauffage isotherme de la pointe, de la distance angulaire $d$ (Fig. 2) séparant deux faces sombres est déterminée ; ceci permet, en répétant l'expérience pour différentes températures, de tracer les courbes en trait fin de la figure 5 .

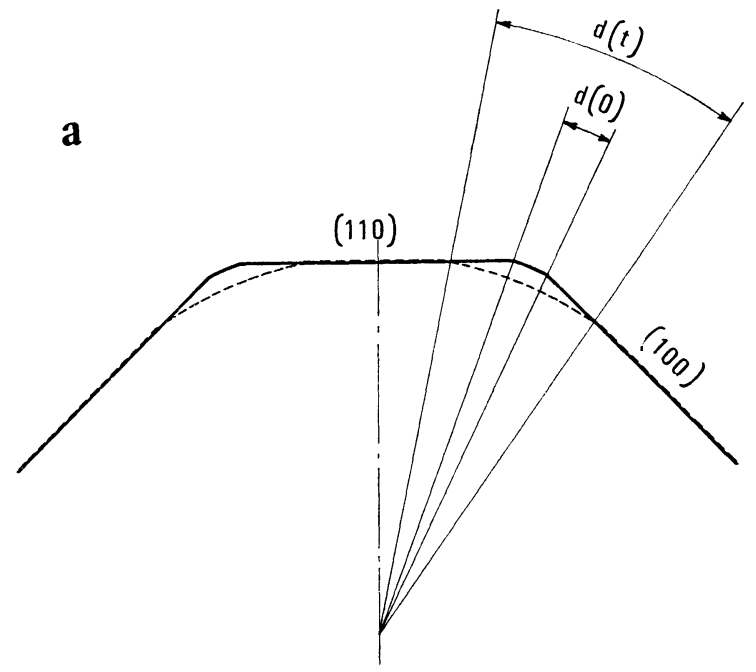

Fig. 2. - Forme de champ ; - - Forme de la pointe à un instant $t$. La mesure est effectuée sur $d$ distance angulaire séparant deux faces sombres. 
- Une simulation graphique du phénomène est effectuée sur ordinateur à l'aide de la relation (7) pour des valeurs arbitraires de $B$ et de $R_{0}$, permettant ainsi de tracer la courbe en trait gras de la figure 5 .

Ce calcul est effectué en se donnant une géométrie donnée (déterminée par l'observation de l'image) de la forme de champ de départ, ce qui permet d'obtenir les coefficients $R_{m}(0)$, et la géométrie de la forme d'équilibre qui permet de calculer les coefficients $\gamma_{m}$.

- La valeur absolue de $D_{\mathrm{s}}$ est alors obtenue pour chaque température en comparant l'abscisse de la courbe expérimentale à celle de la courbe théorique sur la figure 5. En effet, les relations (3) et (7) montrent qu'une fonction quelconque de $R(\theta), d$ en particulier, peut s'exprimer en fonction de la variable $B . t$. $\mathrm{Si}$ l'on trace les graphes correspondant à deux valeurs $T_{1}$ et $T_{2}$ de la température, donc à deux valeurs $B_{1}$ et $B_{2}$ de $B$ en fonction de $\log t$, les courbes obtenues seront parallèles et leurs abscisses différeront d'une quantité égale à $\log B_{1}-\log B_{2}$.

3. Partie expérimentale. - Le dispositif utilisé est un microscope électronique de champ. Après nettoyage de la pointe de tungstène à $2900 \mathrm{~K}$, une forme de champ est obtenue en chauffant la pointe positive en présence d'un champ électrique de l'ordre de $4 \times 10^{7} \mathrm{~V} / \mathrm{cm}$. Cette forme est contrôlée par l'observation microscopique. La température est alors fixée à une valeur donnée pour laquelle la pointe passe, par diffusion de surface, de la forme de champ à la forme d'équilibre (Fig. 2).

$\mathrm{Ce}$ processus est observé et photographié à des intervalles de temps donnés. Le cycle précédent est recommencé pour une série de valeurs de la température de la pointe.

Pour éviter l'influence du champ électrique nécessaire à l'observation sur l'évolution de la forme, nous utilisons un champ pulsé : la largeur à mi-hauteur des impulsions est de $6 \mu \mathrm{s}$, la fréquence de celles-ci est de $25 \mathrm{~Hz}$. Nous avons vérifié, en effectuant des mesures avec et sans champ (dans ce cas l'observation n'est effectuée qu'après refroidissement de la pointe à des températures interdisant la diffusion), que le phénomène n'était pas perturbé, ce qui vérifie bien les études d'autres auteurs $[5,6]$.

L'écran est recouvert à l'intérieur d'une couche d'aluminium transparente aux électrons et opaque à la lumière, permettant ainsi d'éliminer la lumière émise par la pointe à haute température.

Le tirage des clichés est contrôlé avec une cellule photoélectrique de telle sorte que l'éclairement de la zone étudiée ne varie pas, quelle que soit la forme de la pointe au moment du cliché.

Le domaine des températures utilisable est limité à basse température par le temps d'une expérience ( $\mathrm{Si}$ ce temps est très long, les gaz résiduels commencent à contaminer la surface) et, à haute température, par le temps nécessaire aux mesures et à l'établissement d'une température constante. Nous avons utilisé le domaine 1 400-1 $800 \mathrm{~K}$ (durée d'une expérience de 10 à $120 \mathrm{~min}$ ) qui offre l'avantage d'être complémentaire des domaines utilisés par d'autres méthodes $[1,4,5,6](1800$ à $3000 \mathrm{~K})$.

Les mesures sont effectuées dans un vide meilleur que $2 \times 10^{-10}$ torr.

Les températures sont mesurées en déterminant la variation de résistivité de la partie de la boucle de chauffage la plus proche de la pointe. Une correction [16] est effectuée pour tenir compte du gradient de température le long de la pointe. Les distances angulaires $d$ séparant les bords des faces sombres sont mesurées sur les clichés puis corrigées pour tenir compte des variations du grossissement en fonction du rayon de courbure local [17] ; les zones d'étude étant très localisées, nous avons adopté une valeur constante pour la correction pour une zone déterminée.

Le calcul sur ordinateur est effectué en prenant 30 termes pour les séries de Fourier utilisées.

4. Résultats. - Les mesures ont été effectuées entre les faces $(110) \leftrightarrow(100)$ pour deux pointes de rayons différents et entre $(211) \leftrightarrow(100)$, pour une pointe. Dans la direction $(110) \leftrightarrow(100)$, la valeur de $Q_{\mathrm{s}}$ obtenue à l'aide d'un graphe d'Arrhénius (Fig. 3) est égale à $3,8 \mathrm{eV}$. La valeur de $D_{0}$ à $34 \mathrm{~cm}^{2} / \mathrm{s}$. Dans la direction (211) $\leftrightarrow(100), \quad Q_{\mathrm{s}}=3,1 \mathrm{eV} \quad$ et $D_{\mathrm{s}} \simeq 0,3 \mathrm{~cm}^{2} / \mathrm{s}$ (Fig. 4). La valeur de $\gamma_{0}$ utilisée pour le calcul est $\gamma_{0}=2800 \mathrm{erg} / \mathrm{cm}^{2}$.

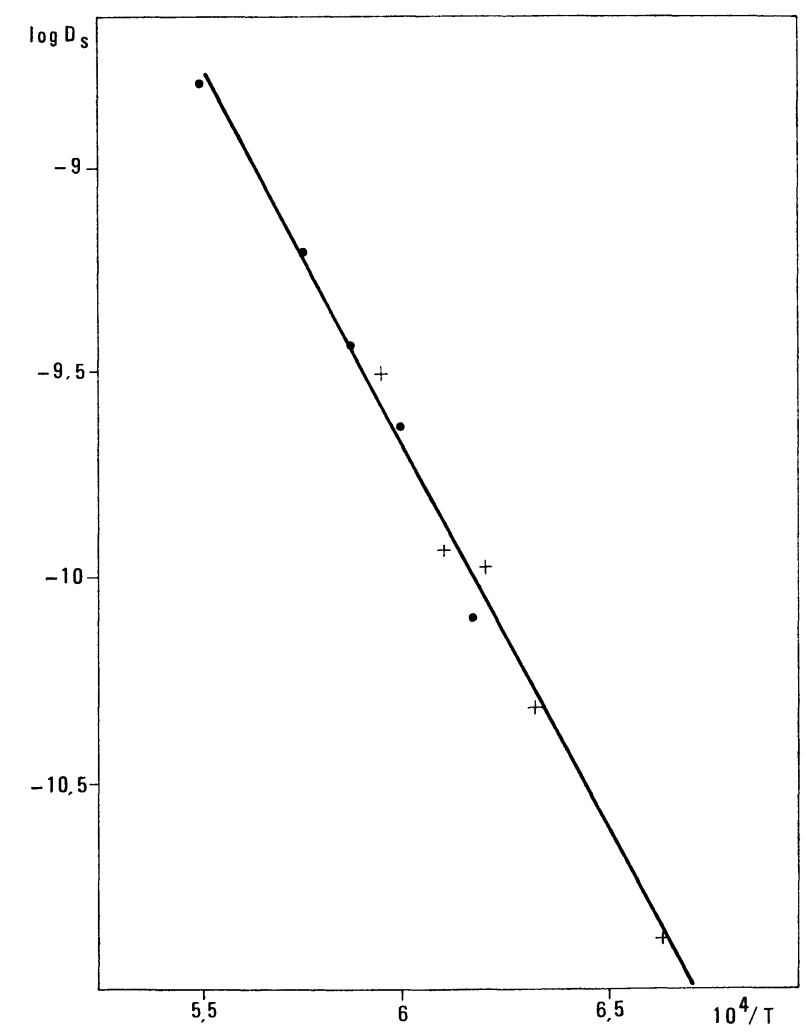

Fig. 3. - $\log D_{\mathrm{s}}$ en fonction de $104 / \mathrm{T}$ pour la zone $(110) \leftrightarrow(100)$ Rayon des pointes : $4 \times 10^{-5} \mathrm{~cm} ;+4,9 \times 10^{-5} \mathrm{~cm}$. 


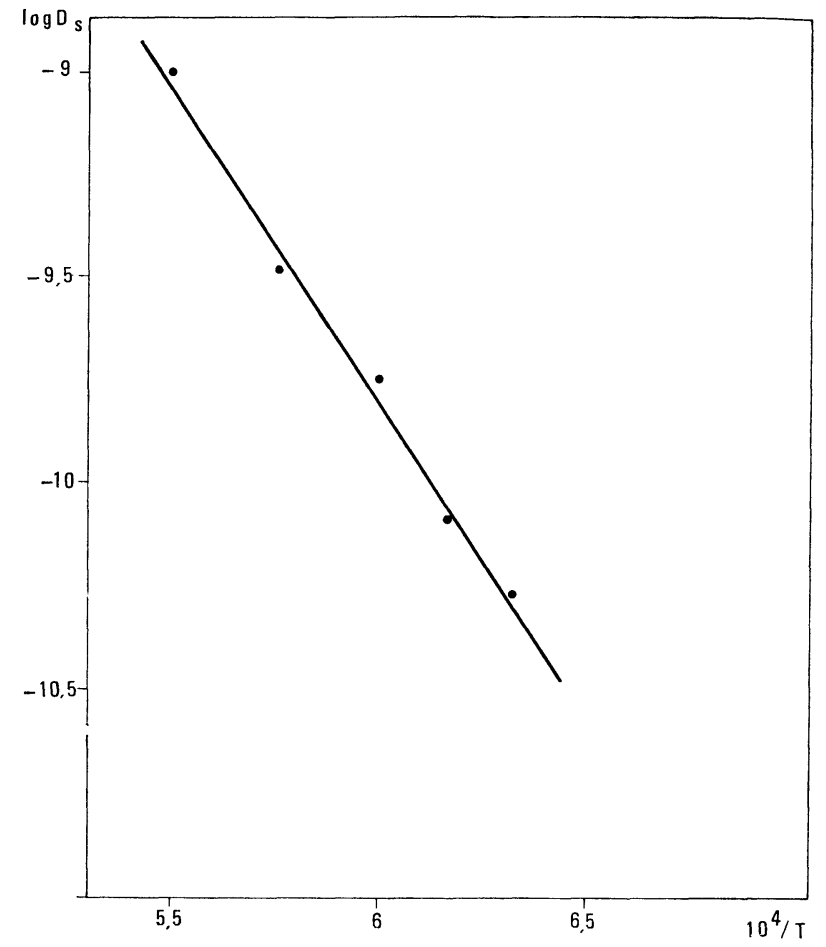

FIG. 4. $-\log D_{\mathrm{s}}$ en fonction de $104 / \mathrm{T}$ pour la zone $(211) \leftrightarrow(100)$ Rayon de la pointe $4 \times 10^{-5} \mathrm{~cm}$.

5. Discussion. - Nous n'avons pas tenu compte des phénomènes de diffusion en volume et d'évaporation condensation. En effet, des études détaillées effectuées par d'autres auteurs [5, 4] montrent que ces phénomènes sont négligeables dans le domaine des températures que nous avons utilisées.

Le calcul du modèle théorique est effectué en négligeant les termes du second ordre du type $R m . R n$, cette approximation est justifiée dans notre cas, le calcul numérique ayant montré que ces produits restaient $\leqslant 10^{-3}$. Nous supposons que l'accord entre les courbes théoriques obtenues et les courbes expérimentales constitue un test de la validité du modèle monodimensionnel, cette comparaison peut s'effectuer sur la figure 5 .

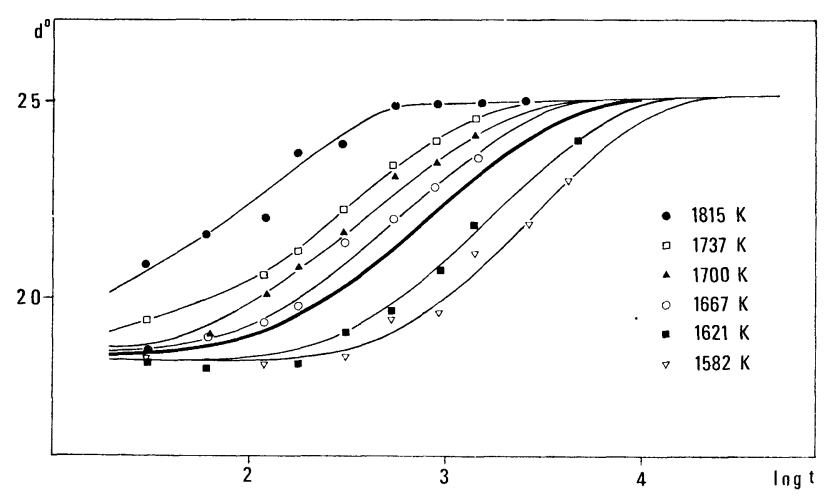

Fig. 5. - Distance angulaire $d$ en fonction de $\log t$. — Courbes. expérimentales pour différentes températures $;===$ courbe théorique pour une valeur arbitraire de $D_{\mathbf{s}}$.
Le bon accord entre le modèle et les résultats expérimentaux est mis en évidence par le parallélisme des courbes.

De plus le fait que les points obtenus sur les figures 3 et 4 sont raisonnablement alignés constitue un contrôle supplémentaire.

Si un cristal change de forme par diffusion de surface, le nombre de types de déplacements d'un atome entre deux positions voisines est assez grand. Cependant, le nombre de types des déplacements décisifs pour le changement de forme est assez faible; la méthode décrite ne permet de mesurer le coefficient $D_{\mathrm{s}}$ que pour ces déplacements.

La notion $(110) \leftrightarrow(100)$ (ou $(211) \leftrightarrow(100)$ ) peut être précisée. Il s'agit des déplacements des atomes des faces situées entre (110) et (100), c'est-à-dire en particulier de (210) vers (110) et (100) (Fig. 6).

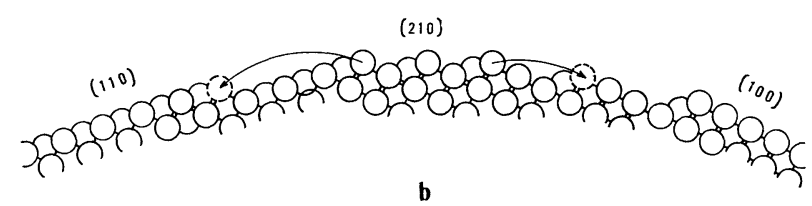

Fig. 6. - Déplacement par autodiffusion de surface des atomes entre (110) et (100).

Il aurait été intéressant de pouvoir comparer nos résultats à ceux de Bowden et Singer [18] et Allen [19] qui tiennent compte de l'orientation du cristal, mais ces auteurs ont travaillé dans la gamme des hautes températures, domaine dans lequel la valeur de $Q_{\mathrm{s}}$ est plus élevée qu'à basse température.

La comparaison des valeurs que nous avons obtenues avec celles provenant de méthodes qui ne tiennent pas compte de l'anisotropie de la diffusion $(3,1 \mathrm{eV}[12,4,5,9])$ est moins significative. Cependant, on peut noter que l'ordre de grandeur des valeurs trouvées est le même.

Les valeurs de $Q_{\text {s }}$ mesurées devraient pouvoir être comparées avec des valeurs théoriques. Des valeurs de ce type ont été calculées jusqu'à présent avec un modèle simplifié par Drechsler et Vanselow [10]. (Les calculs plus élaborés de Wynblatt et Gjostein [20] ne concernent que des déplacements d'adatomes sur des faces idéales mais pas le passage des marches.) Pour le déplacement du type (011) $\leftrightarrow(001)$ (Fig. 6), le calcul donne $Q_{\mathrm{s}}=4,38 \mathrm{eV}$ à comparer avec notre valeur mesurée de $3,8 \mathrm{eV}$. Pour le déplacement $(112) \leftrightarrow(001)$ aucune valeur théorique n'a été calculée, mais une estimation qualitative montre que la valeur de $Q_{\mathrm{s}}$ doit être inférieure à la précédente, ce qui est bien vérifié par nos mesures. Dans la direction $(110) \leftrightarrow(211)$ les résultats ne sont pas exploitables à l'heure actuelle en raison du manque de précision des résultats. Ce manque de précision est principalement dû au fait de zones à très fort rayon de courbure entre les deux faces qui se traduisent par des taches très fortement éclairées sur l'image; de toutes façons, dans ce cas particulier, l'application 
d'un modèle de diffusion monodimensionnelle paraît être douteuse.

La précision obtenue pour $Q_{\mathrm{s}}$ est de l'ordre de $0,1 \mathrm{eV}$. Pour $D_{0}$, l'erreur la plus importante provient du terme $R_{0}^{4} ; R_{0}$ étant connu avec une précision de $15 \%$, ceci entraîne une erreur sur $D_{0}$ de $60 \%$ à laquelle s'ajoutent les erreurs sur les températures ou les distances angulaires mesurées. Nous estimons que les résultats obtenus doivent pour tenir compte de l'erreur être multipliés par un facteur $\mathrm{e}^{0 \pm, 7}$.

Cette erreur est relativement élevée, cependant elle doit être rapprochée des valeurs de $D_{0}$ trouvées dans la littérature qui varient souvent de plusieurs ordres de grandeur.
Des améliorations de la technique de mesure sont actuellement en cours pour différencier les déplacements du type $(210) \leftrightarrow(100)$ et $(210) \leftrightarrow(110)$ (Fig. 6). Ces mesures devraient permettre des comparaisons plus faciles avec les résultats théoriques existants ou en cours de calcul.

6. Conclusion. - Nous avons mis au point une méthode de mesure rapide des coefficients de l'équation d'autodiffusion de surface dans des directions (zones) cristallographiques déterminées, dans un domaine de température complémentaire des méthodes habituellement utilisées. La méthode permet un contrôle continu de la propreté de la surface.

\section{Bibliographie}

[1] Müller, E. W., Z. Phys. 126 (1949) 642.

[2] Ehrlich, G. and Hudda, F. G., J. Chem. Phys. 44 (1966) 1039.

[3] Basset, D. W. and Parsley, M. J., J. Phys. D : Appl. Phys. 3 (1970) 707

[4] Piquet, A., Thèse, Univ. Claude-Bernard, Lyon (1973).

[5] Barbour, J. P., Charbonnier, F. H., Dolan, W. W., Dyke, W. P., Martin, E. E. and Trolan, J. K., Phys. Rev. 117 (1960) 1452.

[6] WARNER, H. R., Thesis, Carnegie Institute of Tech. (1967).

[7] Bettler, P. S. and Barnes, G., Surf. Sci. 10 (1968) 165.

[8] Vu Thien, B., Piquet, A., Roux, H., Uzan, R., Drechsler, M., Surf. Sci. 25 (1971) 348.

[9] Piquet, A., Roux, H., Vu Thien, B., Uzan, R., DrechsLER, M., Surf. Sci., sous presse.

[10] Drechsler, M. und Vanselow, R., Z. Kristallogr. 107 (1956) 161
[11] Sokolkaya, L. L., Sov. Phys. Techn. Phys. 1 (1956) 1147.

[12] Pichaud, M. et Drechsler, M., Surf. Sci. 32 (1972) 341.

[13] Pichaud, M. et Drechsler, M., Surf. Sci. 36 (1973) 813.

[14] Melmed, A. J., J. Appl. Phys. 38 (1967) 1885.

[15] Guostein, M. A., cité par Bonzel, H. P., Structure and properties of Metal Surfaces (Ed. Maruzen, Co. Japan) 1972.

[16] Pichaud, M., Müller, A. and Drechsler, M., Surf. Sci. 26 (1971) 14.

[17] Müller, A. und Drechsler, M., Surf. Sci. 13 (1969) 471.

[18] Bowden, F. P. and Singer, K. E., Nature 222 (1969) 977.

[19] Allen, B. C., Trans. AIME 236 (1966) 915.

[20] Wynblatt, P. and Guostein, M. A., Surf. Sci. 12 (1968) 109.

[21] Nichols, F. A. and Mullins, W. W., J. Appl. Phys. 36 (1965). 